\title{
Significance of Neutrophil Gelatinase-Associated Lipocalin as a Biomarker for the Diagnosis of Diabetic Kidney Disease: A Systematic Review and Meta-Analysis
}

\author{
Peng He ${ }^{a} \quad$ Ming Bai ${ }^{a} \quad$ Jin-ping Hu ${ }^{a} \quad$ Chen Dong ${ }^{b}$ Shiren Sun ${ }^{a}$ Chen Huang ${ }^{a}$ \\ a Department of Nephrology, Xijin Hospital, The Fourth Military Medical University, Xi'an, China; \\ ${ }^{b}$ Graduate School, The Fourth Military Medical University, Xi'an, China
}

\section{Keywords}

Neutrophil gelatinase-associated lipocalin · Diabetic kidney disease $\cdot$ Meta-analysis

\begin{abstract}
Background: Neutrophil gelatinase-associated lipocalin (NGAL) is a promising marker for the diagnosis of diabetic kidney disease (DKD), but its utility is currently debated. This meta-analysis aims to evaluate the diagnostic value of NGAL for DKD. Method: MEDLINE, Embase, Cochrane Library, CNKI, and CBM databases were searched up to April 13, 2019. In bivariate random-effect models, the diagnostic performance of NGAL for DKD was assessed using pooled estimates of sensitivity, specificity, likelihood ratio, diagnostic odds ratio, and hierarchical summary receiver-operating characteristic analysis. Results: Nineteen studies were eligible for the meta-analysis. Serum NGAL had a pooled sensitivity and specificity of 0.79 (95\% confidence intervals [Cl] 0.60-0.91) and 0.87 (0.75-0.93) (7 studies, 1,238 patients). The pooled positive likelihood ratio (LR+) and negative likelihood ratio (LR-) were 5.97 (3.03-11.76) and $0.24(0.11-0.51)$. For urine NGAL, the pooled sensitivity, specificity, LR+, and LR- were 0.85 $(0.74-0.91), 0.74(0.57-0.86), 3.26(1.87-5.67)$, and $0.21(0.12-0.35)$, respectively (10 studies, 1,369 patients). The pooled sensitivity and specificity for kidney disease in normoalbuminuric patients with diabetes was $0.90(0.82-0.95)$ and $0.97(0.90-0.99)$ for both serum NGAL and 0.94 (0.87-0.98) and 0.90 (0.81-0.96) for urine NGAL (4 studies, 221 patients). NGAL appeared to perform similarly in subgroup analysis. Conclusion: The meta-analysis has shown that NGAL may be useful for DKD classification and also has a potential diagnostic value for normoalbuminuric kidney disease. Large-scale prospective studies are required to clarify its role in the diagnosis and risk stratification of patients with DKD.




\section{Kidney \\ Blood Pressure \\ Research}

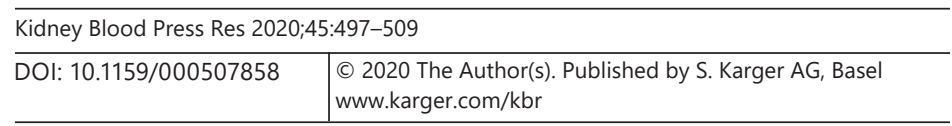

He et al.: Value of NGAL for DKD Diagnosis

\section{Introduction}

Diabetic kidney disease (DKD) is one of the most important microvascular complications of diabetes mellitus (DM). Approximately $20-40 \%$ of patients with DKD develop end-stage renal disease requiring renal replacement therapy $[1,2]$. The urinary albumin-to-creatinine ratio (UACR) and formulas to assess estimated glomerular filtration rate (eGFR) are parameters currently used to assess the presence and progression of DKD. However, they are imprecise, do not directly measure histological injury of the kidneys, and are relatively insensitive to small changes in renal function [3]. The gold standard for DKD diagnosis is renal biopsy. However, this method is both invasive and labor intensive. Therefore, it is necessary to look for more sensitive and noninvasive markers in this field.

Studies have repeatedly confirmed that renal tubulointerstitial injury plays a crucial role in the progression of DKD [3, 4]. Neutrophil gelatinase-associated lipocalin (NGAL) is a structural tubular marker (with a weight of $25 \mathrm{kDa}$ ) belonging to the lipocalin superfamily, which is extensively increased in the serum or urine of patients within a few hours after ischemia reperfusion injury. Although both preclinical $[5,6]$ and clinical cohort studies $[7,8]$ have shown a correlation between NGAL levels and the severity of albuminuria or renal impairment, the diagnostic power of NGAL for DKD has yet to be elucidated. To address these issues, we conducted a systemic review and meta-analysis of observational studies published through April 13, 2019.

\section{Methods}

Data Sources and Search Strategy

Our study adhered to the Preferred Reporting Items for Systematic Reviews and MetaAnalyses (PRISMA) guidelines [9] and the meta-analysis of observational studies in epidemiology (MOOSE) guidelines [10]. Two researchers (P. H. and C.D.) independently conducted a comprehensive literature search in MEDLINE (via PubMed), Embase, Cochrane Library, China National Knowledge Infrastructure (CNKI), and Chinese Biology Medical (CBM) databases through April 13, 2019, without language restrictions. Search terms included neutrophil gelatinase-associated lipocalin, NGAL, diabetic nephropathy, and diabetic kidney disease, and the strategy is detailed in online supplementary item S1 (for all online suppl. material, see www. karger.com/doi/10.1159/000507858). We also reviewed the reference lists for other relevant studies.

\section{Inclusion and Exclusion Criteria}

After removing duplicate studies, 2 researchers (P.H. and C.D.) independently performed a preliminary screening according to the titles and abstracts and subsequently the full texts. Any disagreement on eligibility was resolved through discussion and consensus with the arbitrator (C.H.). The inclusion criteria were as follows: (a) human observational studies including males and females; (b) sample size no less than 30; (c) plasma/serum (sNGAL) or urine NGAL (UNGAL) tested for DKD diagnosis using the receiver-operating characteristic curve (ROC) analysis; (d) the use of the American Diabetes Association (ADA) Standards of Medical Care in Diabetes $[11,12] /$ the Guidelines for the Prevention and Treatment of Type 2 DM (T2DM) in China [13] as reference standards for defining type 1 DM (T1DM)/T2DM; and (e) the use of the Kidney Disease Outcomes Quality Initiative (KDOQI) [14]/Kidney Disease: Improving Global Outcomes (KDIGO) [15]/Expert Consensus on Prevention and Treatment of DKD in China as the diagnostic criteria for DKD [16]. The exclusion criteria were as follows: (a) reviews; (b) animal studies; (c) conference abstracts; (d) poster presentations; (e) no diagnostic experimental methods; and (f) studies with insufficient data. 


\section{Research}

He et al.: Value of NGAL for DKD Diagnosis

Fig. 1. Flowchart of literature

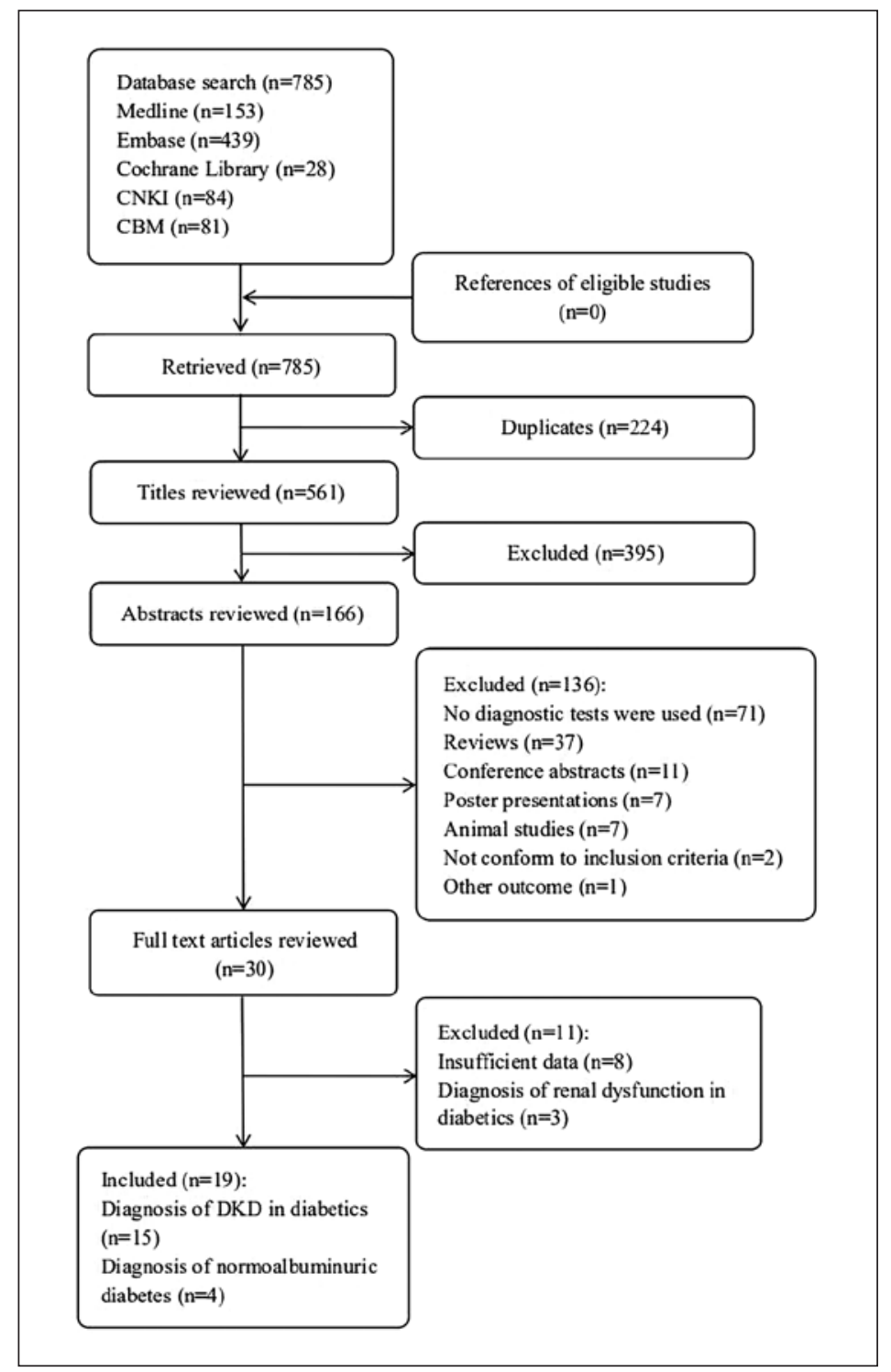
identification and selection.

\section{Data Extraction and Quality Assessment}

Two researchers (P.H. and J.H.) extracted or calculated prespecified information from each study independently and produced standardized tables. The variables extracted included first author, year of publication, study location, design, diagnostic criteria, target group, sample size, mean age, specimen, NGAL assay, and storage temperature. Diagnostic test parameters included sensitivity, specificity, and the optimal cutoff value. The absolute numbers of true positives (TPs), false positives (FPs), true negatives (TNs), and false negatives (FNs) were extracted directly or were calculated to construct $2 \times 2$ contingency tables. If the table could not be reconstructed due to insufficient data, the authors were contacted via email to ask whether they could share the unpublished data. If there was no response, the corresponding study would not be included. The methodological quality of eligible studies was evaluated by the Quality Assessment of Diagnostic Accuracy Studies 2 (QUADAS-2) tool [17]. Operational definitions for high, low, and unclear risk of bias were developed for each of the 14 QUADAS-2 domains. Each study was reviewed by P.H. and J.H., and then rated as high, low, or unclear risk for each domain. 
Table 1. Summary of the included studies

\begin{tabular}{|c|c|c|c|c|c|c|c|c|c|}
\hline First author, year & Location & $\begin{array}{l}\text { Diagnostic } \\
\text { criteria }\end{array}$ & $\begin{array}{l}\text { Target group, } \\
\text { cases/controls }\end{array}$ & $\begin{array}{l}\text { Total } \\
n\end{array}$ & $\begin{array}{l}\text { Cases/ } \\
\text { controls, } \\
n\end{array}$ & $\begin{array}{l}\text { Mean age } \\
\text { cases/ } \\
\text { controls }\end{array}$ & Specimen & Storage & $\begin{array}{l}\text { NGAL } \\
\text { assay }\end{array}$ \\
\hline Sueud [20], 2019 & Australia & $\begin{array}{l}\text { ADA } \\
\text { eGFR/uACR }\end{array}$ & $\begin{array}{l}\text { DKD/ } \\
\text { non-DKD }\end{array}$ & 90 & $75 / 15$ & $54.3 / 53.3$ & Urine & NR & ELISA \\
\hline Zeng [29], 2017 & China & $\begin{array}{l}\text { ADA } \\
\text { eGFR/uACR }\end{array}$ & $\begin{array}{l}\text { DKD/ } \\
\text { non-DKD }\end{array}$ & 146 & $42 / 104$ & $55.7 / 57.6$ & Urine & $-80^{\circ} \mathrm{C}$ & ELISA \\
\hline Zylka [22], 2018 & Poland & $\begin{array}{l}\text { ADA } \\
\text { uACR }\end{array}$ & $\mathrm{MI} / \mathrm{NO}$ & 80 & $19 / 61$ & $59 / 67$ & $\begin{array}{l}\text { Serum } \\
\text { Urine }\end{array}$ & $-80^{\circ} \mathrm{C}$ & $\begin{array}{l}\text { ELISA } \\
\text { CLIA }\end{array}$ \\
\hline Vijay [23], 2018 & India & $\begin{array}{l}\text { ADA } \\
\text { uACR }\end{array}$ & $\mathrm{MI} / \mathrm{NO}$ & 126 & $63 / 63$ & $54.3 / 49.2$ & Urine & $-20^{\circ} \mathrm{C}$ & ELISA \\
\hline Kaul [24], 2018 & India & $\begin{array}{l}\text { ADA } \\
\text { UACR }\end{array}$ & $\mathrm{MA}+\mathrm{MI} / \mathrm{NO}$ & 144 & $108 / 36$ & $57 / 53.5$ & $\begin{array}{l}\text { Serum } \\
\text { Urine }\end{array}$ & $-80^{\circ} \mathrm{C}$ & ELISA \\
\hline Motawi [25], 2017 & Egypt & $\begin{array}{l}\text { ADA } \\
\text { uACR }\end{array}$ & $\mathrm{MI} / \mathrm{NO}$ & 50 & $25 / 25$ & $50.5 / 48.8$ & Serum & NR & ELISA \\
\hline Mahfouz [32], 2016 & $\begin{array}{l}\text { Saudi } \\
\text { Arabia }\end{array}$ & $\begin{array}{l}\text { ADA } \\
\text { uACR }\end{array}$ & $\mathrm{MA}+\mathrm{MI} / \mathrm{NO}$ & 150 & $100 / 50$ & $54.7 / 55.7$ & Serum & $-80^{\circ} \mathrm{C}$ & ELISA \\
\hline Assal [34], 2013 & Egypt & $\begin{array}{l}\text { ADA } \\
\text { uACR }\end{array}$ & $\mathrm{MI} / \mathrm{NO}$ & 45 & $25 / 20$ & $52.9 / 51.3$ & Urine & $-20^{\circ} \mathrm{C}$ & ELISA \\
\hline Liu [21], 2019 & China & $\begin{array}{l}\text { CSE } \\
\text { uACR }\end{array}$ & $\mathrm{MA}+\mathrm{MI} / \mathrm{NO}$ & 376 & $181 / 195$ & 58 & Serum & NR & TIIA \\
\hline Zhong [27], 2018 & China & $\begin{array}{l}\text { ADA } \\
\text { uAER }\end{array}$ & $\mathrm{MA}+\mathrm{MI} / \mathrm{NO}$ & 154 & $96 / 58$ & $56.6 / 55.2$ & Serum & NR & ELISA \\
\hline Chen [28], 2018 & China & $\begin{array}{l}\text { CSE } \\
\text { uAER }\end{array}$ & $\mathrm{MA}+\mathrm{MI} / \mathrm{NO}$ & 300 & $120 / 180$ & NR & Urine & $-20^{\circ} \mathrm{C}$ & TIIA \\
\hline Yang [31], 2017 & China & $\begin{array}{l}\text { CSE } \\
\text { uACR }\end{array}$ & $\mathrm{MA}+\mathrm{MI} / \mathrm{NO}$ & 284 & $170 / 114$ & 51.3 & Serum & NR & ELISA \\
\hline Wang [26], 2018 & China & $\begin{array}{l}\text { CSE } \\
\text { uAER }\end{array}$ & $\mathrm{MI} / \mathrm{NO}$ & 340 & $210 / 130$ & $61.7 / 61.2$ & Urine & $-80^{\circ} \mathrm{C}$ & ELISA \\
\hline Dayem [30], 2017 & Egypt & $\begin{array}{l}\text { ADA } \\
\text { uACR }\end{array}$ & $\mathrm{MA}+\mathrm{MI} / \mathrm{NO}$ & 48 & $20 / 28$ & 19.2 & Urine & $-80^{\circ} \mathrm{C}$ & ELISA \\
\hline Hafez [33], 2015 & Egypt & $\begin{array}{l}\text { ADA } \\
\text { uACR }\end{array}$ & $\mathrm{MI} / \mathrm{NO}$ & 50 & $12 / 38$ & 13.8 & Urine & $-20^{\circ} \mathrm{C}$ & TIIA \\
\hline Hosny [35], 2018 & Egypt & $\begin{array}{l}\text { ADA } \\
\text { uACR }\end{array}$ & $\mathrm{NO} / \mathrm{HC}$ & 40 & $20 / 20$ & $58.2 / 56.7$ & Urine & NR & ELISA \\
\hline Shen [36], 2016 & China & $\begin{array}{l}\text { CSE } \\
\text { uAER }\end{array}$ & $\mathrm{NO} / \mathrm{HC}$ & 62 & $27 / 35$ & $63.2 / 63.7$ & Serum & $-80^{\circ} \mathrm{C}$ & ELISA \\
\hline Lacquaniti [37], 2013 & Italy & $\begin{array}{l}\text { ADA } \\
\text { uACR }\end{array}$ & $\mathrm{NO} / \mathrm{HC}$ & 85 & $50 / 35$ & $36.9 / 34.9$ & $\begin{array}{l}\text { Serum } \\
\text { Urine }\end{array}$ & $-80^{\circ} \mathrm{C}$ & ELISA \\
\hline Bolignano [8], 2009 & Italy & $\begin{array}{l}\text { ADA } \\
\text { uACR }\end{array}$ & $\mathrm{NO} / \mathrm{HC}$ & 34 & $16 / 18$ & $49 / 52$ & $\begin{array}{l}\text { Serum } \\
\text { Urine }\end{array}$ & $-80^{\circ} \mathrm{C}$ & ELISA \\
\hline
\end{tabular}

ADA, American Diabetic Association criteria; CLIA, chemiluminescent immunoassay; CSE, Chinese Society of Endocrinology criteria; DKD, diabetic kidney disease; eGFR, estimated glomerular filtration rate ( $\left.\leq 60 \mathrm{~mL} / \mathrm{min} / 1.73 \mathrm{~m}^{2}\right)$; ELISA, enzyme-linked immunosorbent assay; HC, healthy control; MA, macroalbuminuria; MI, microalbuminuria; NGAL, neutrophil gelatinase-associated lipocalin; NO, normoalbuminuria; NR, not reported; TIIA, turbidimetric inhibition immunoassay; uACR, urinary albumin creatinine ratio ( $\geq 30 \mathrm{mg} / \mathrm{g}$ ); uAER, urinary albumin excretion rate $(\geq 30 \mathrm{mg} / 24 \mathrm{~h})$. 


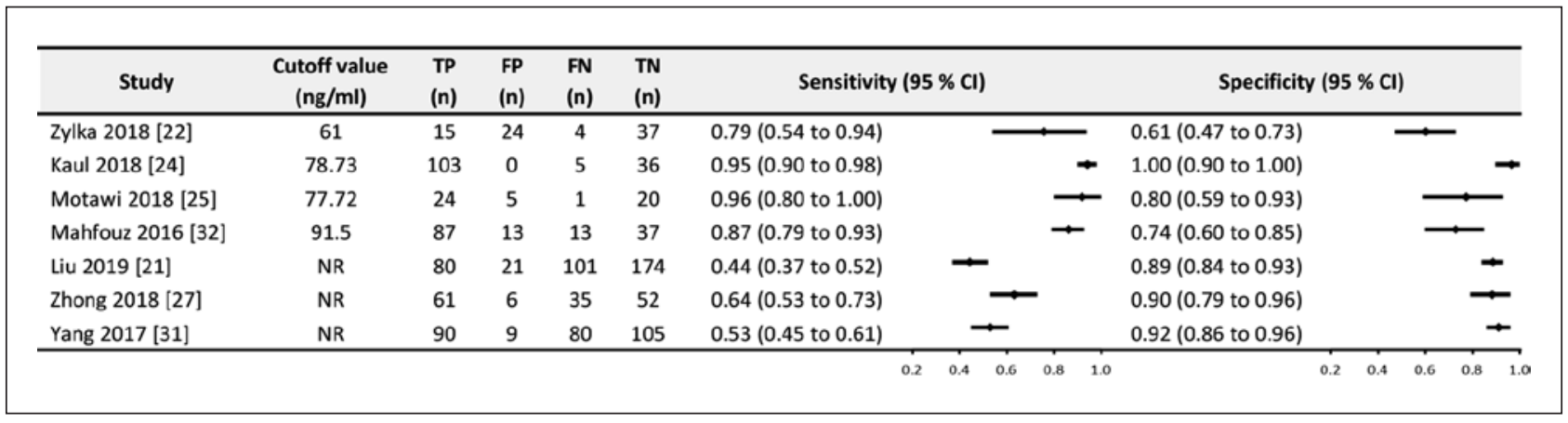

Fig. 2. Forest plots of the sensitivity and specificity of serum neutrophil gelatinase-associated lipocalin for the diagnosis of diabetic kidney disease. CI, confidence interval; FP, false positive; FN, false negative; NR, not reported; $\mathrm{TP}$, true positive; $\mathrm{TN}$, true negative.

\section{Statistical Analysis}

Our meta-analysis was performed with the "metandi" command in Stata (version 15.0) software. Because of foreseeable and significant heterogeneity in test accuracy between studies, a bivariate random-effect model was selected that provided an estimate of the average accuracy of the test and described the variability in the effect $[18,19]$. This method not only preserves the two-dimensional characteristics of the original data but also comprehensively estimates the negative correlation between sensitivity and specificity. In the bivariate model, we calculated pooled sensitivity, specificity, likelihood ratios, and diagnostic odds ratio (DOR) and created the hierarchical summary ROC curve (HSROC). We also performed subgroup analyses by restricting the analysis to studies with the following characteristics: micro- or macroalbuminuria, T1DM or T2DM, location, number of cases, test method, and storage temperature. Subject to the number of included studies, Meta-DiSc (version 1.4) software was used to evaluate the diagnostic value of NGAL for normoalbuminuric diabetes.

\section{Results}

\section{Search Results and Study Characteristics}

We retrieved 785 articles from the databases, and 561 articles remained after eliminating duplicate articles. After reviewing titles and abstracts, 531 were excluded after implementing the eligibility criteria. Of the 30 studies included in the full-text evaluation, 8 were excluded for insufficient data, and 3 were excluded for renal decline in the diabetic patients. Finally, 19 studies were included in the meta-analysis [8, 20-37] (Fig. 1).

Table 1 summarizes the studies that were analyzed. Eleven cross-sectional, 5 retrospective, and 3 prospective studies from 7 countries with a cumulative sample size of 2,604 were reviewed. Fifteen studies attempted to evaluate the diagnostic value of NGAL for DKD. eGFR $\leq 60 \mathrm{~mL} / \mathrm{min} / 1.73 \mathrm{~m}^{2}$ or UACR $>30 \mathrm{mg} / \mathrm{g}$ was used as the diagnostic threshold. Four studies aimed to explore the diagnostic accuracy of NGAL to discriminate between normoalbuminuric diabetics and healthy controls.

\section{Quality Assessment}

The overall impression of literature quality is presented in online supplementary Figure S2. Due to the lack of detailed inclusion and exclusion criteria in 2 studies, there were certain risks in case selection. As mentioned above, the reference standards and target groups of eligible studies were slightly different, which may lead to selection bias. None of the 19 studies 


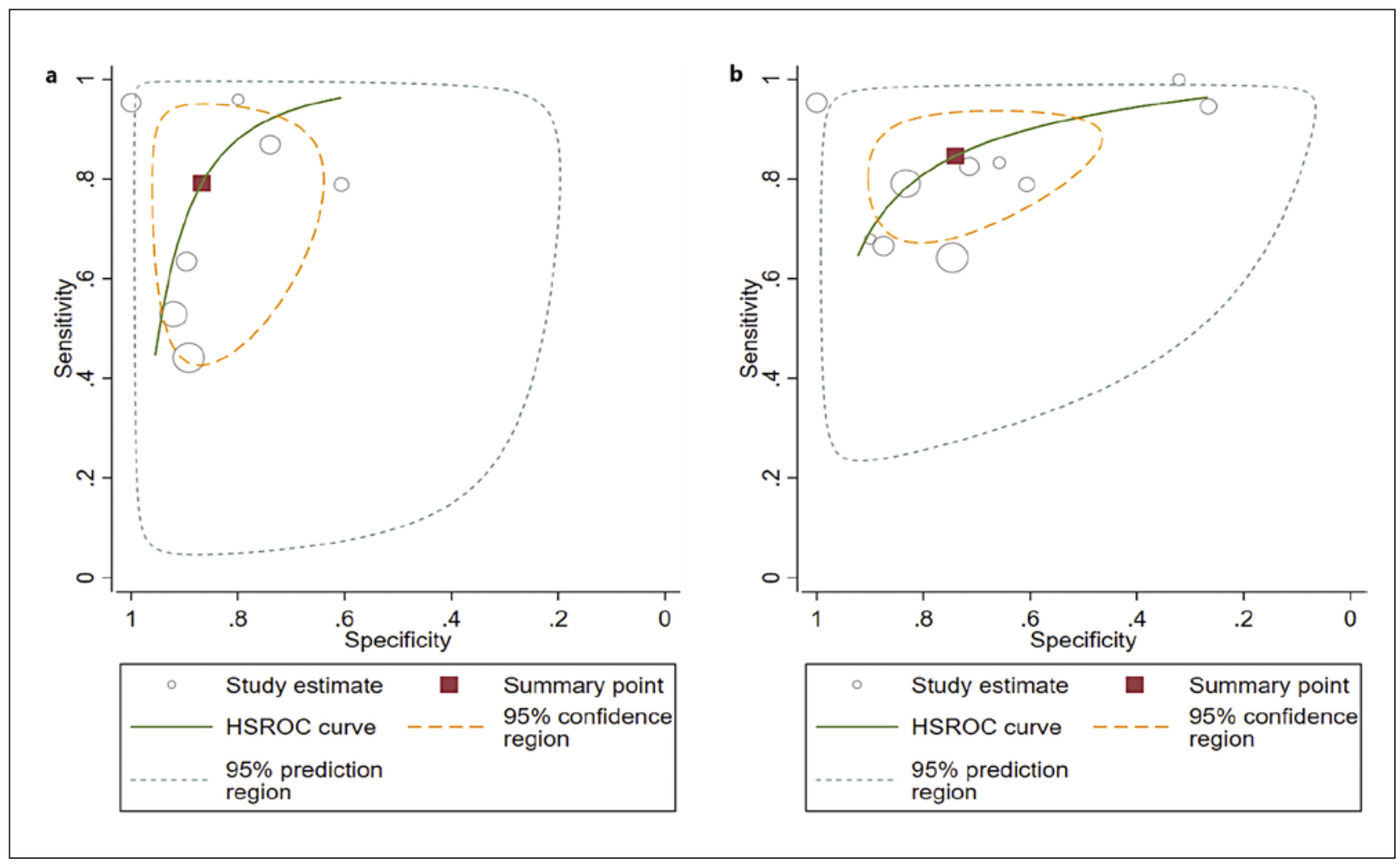

Fig. 3. Hierarchical summary receiver-operating characteristic curve (HSROCs) of neutrophil gelatinaseassociated lipocalin (NGAL) for diabetic kidney disease (DKD) diagnosis. a HSROC of serum NGAL for DKD diagnosis. b HSROC of urine NGAL for DKD diagnosis.

made it clear whether the results of the trials to be evaluated were interpreted without knowing the results of the reference trials, and we could not determine whether the studies complied with the blinding method. This might lead to measurement bias and exaggerate the diagnostic performance.

\section{Diagnosis of DKD using SNGAL}

Seven studies, including 1,238 patients, investigated the diagnostic accuracy of sNGAL for DKD. The key parameters of the diagnostic tests are summarized in Figure 2. In the bivariate model, the pooled sensitivity and specificity were 0.79 (95\% confidence interval [CI]: 0.60-0.91) and 0.87 (0.75-0.93), respectively. The pooled positive likelihood ratio (LR+) was 5.97 (3.0311.76), the negative likelihood ratio (LR-) was 0.24 (0.11-0.51), and the DOR value was 24.82 (7.54-81.66). HSROC was conducted to reflect the classified accuracy (Fig. 3). In subgroup analyses (Table 2), 5 studies involving 1,108 patients enrolled patients with both micro- and macroalbuminuria in diabetes, with a pooled sensitivity, specificity, and LR+ of $0.74(0.50-0.89)$, 0.91 (0.81-0.96), and 8.02 (3.36-19.14), respectively. Results were similar among studies enrolling 100 or more patients or using the enzyme-linked immunosorbent assay (ELISA).

\section{Diagnosis of DKD using uNGAL}

The uNGAL assay was performed in 10 studies involving 1,369 patients (Fig. 4). The pooled sensitivity was $0.85(0.74-0.91)$, specificity was $0.74(0.57-0.86)$, LR+ was 3.26 (1.875.67), LR- was 0.21 (0.12-0.35), and DOR value was 15.73 (6.75-36.65). Subgroup analyses (Table 2) identified 5 studies involving 641 diabetic patients with microalbuminuria. In this 
Kidney

Blood Pressure

Research

\begin{tabular}{l|l}
\hline Kidney Blood Press Res 2020:45:497-509 \\
\hline DOI: 10.1159/000507858 & $\begin{array}{l}\text { @ 2020 The Author(s). Published by S. Karger AG, Basel } \\
\text { www.karger.com/kbr }\end{array}$ \\
\hline
\end{tabular}

He et al.: Value of NGAL for DKD Diagnosis

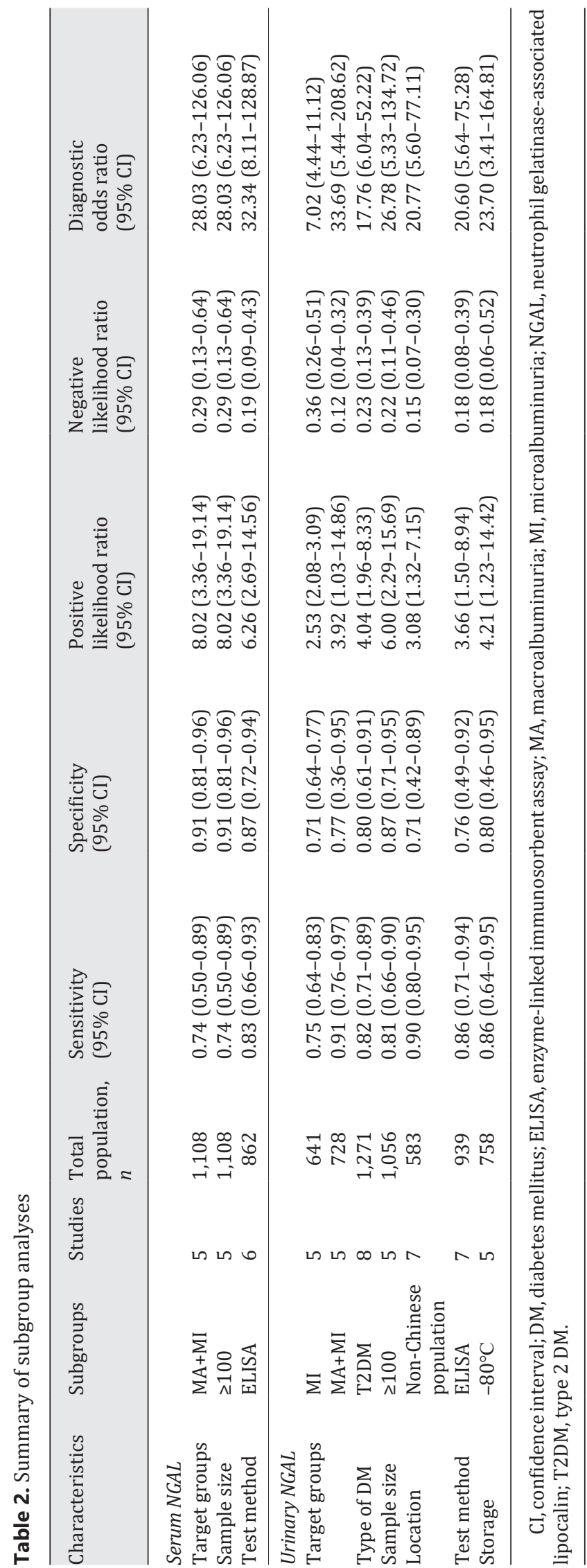




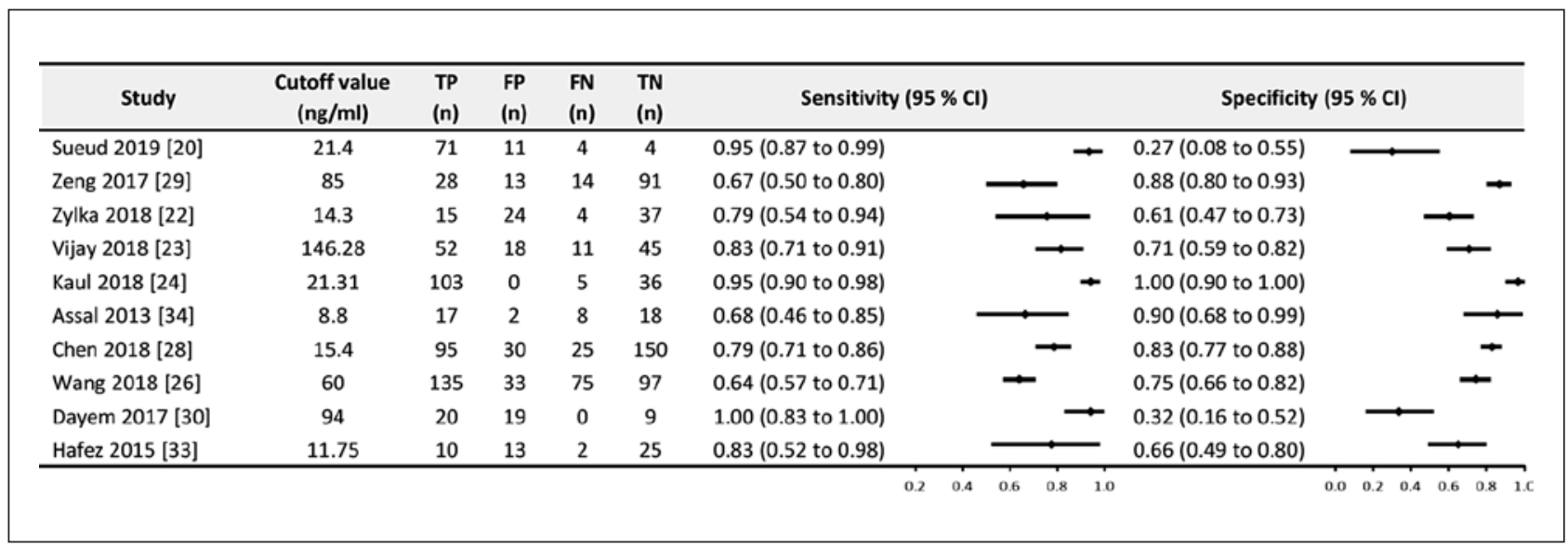

Fig. 4. Forest plots of the sensitivity and specificity of urine neutrophil gelatinase-associated lipocalin for diabetic kidney disease diagnosis. CI, confidence interval; FP, false positive; FN, false negative; TP, true positive; $\mathrm{TN}$, true negative.

population, the pooled sensitivity was $0.75(0.64-0.83)$, specificity was $0.71(0.64-0.77)$, and LR+ was 2.53 (2.08-3.09). A further 5 studies included 492 diabetic patients with both microand macroalbuminuria, and the pooled sensitivity, specificity, and LR+ were 0.91 (0.76-0.97), 0.77 (0.36-0.95), and 3.92 (1.03-14.86), respectively. Similar test performance was noted in studies restricted to those with type 2 diabetes, enrolling 100 or more patients, using ELISA, storage of the sample at $-80^{\circ} \mathrm{C}$, or conducted outside of China (Table 2).

\section{Diagnosis of Normoalbuminuric Diabetes}

Four studies including 221 subjects (normoalbuminuric diabetics and healthy controls) aimed to identify diabetic patients with normal albumin excretion (online suppl. Table S3). For sNGAL, pooled sensitivity and specificity were $0.90(0.82-0.95)$ and $0.97(0.90-0.99)$, respectively. The pooled area under the curve (AUC) was 0.973. For UNGAL, the pooled sensitivity, specificity, and AUC were 0.94 (0.87-0.98), 0.90 (0.81-0.96), and 0.983, respectively (online suppl. Fig. S4).

\section{Diagnosis of Renal Decline}

Three studies estimated the classified value of NGAL for renal decline but lacked consistent definitions for meta-analysis. Bacci et al. [38] evaluated the value of NGAL in screening renal dysfunction (defined as eGFR $<60 \mathrm{~mL} / \mathrm{min} / 1.73 \mathrm{~m}^{2}$ ) in $90 \mathrm{~T} 2 \mathrm{DM}$ patients. In terms of diagnostic accuracy, the AUCs were $0.66(0.53-0.78)$ for UACR, $0.62(0.51-0.71)$ for sNGAL, and $0.53(0.42-0.64)$ for uNGAL. Satirapoj et al. [39] conducted a follow-up study on 303 patients. The UNGAL-to-creatinine ratio (UNCR) was used to identify T2DM patients whose eGFR decreased by more than $25 \%$ from baseline in 1 year. ROC analysis showed an AUC of 0.64 (0.56-0.72). Sensitivity and specificity were 0.68 and 0.51 , respectively (optimal cutoff level $772 \mathrm{ng} / \mathrm{g}$ ). Yürük Yildirim et al. [40], who recruited 76 T1DM and 35 healthy individuals, found UNGAL had an AUC of 0.95 for the diagnosis of DKD. The optimal cutoff value was 36.3 $\mathrm{ng} / \mathrm{ml}$ (sensitivity 0.95 , specificity 0.94 ).

\section{Studies Unable to be Included in Meta-Analysis}

Seven studies [41-47], including 697 patients, did not provide sufficient information to permit construction of the $2 \times 2$ contingency tables. These studies were broadly in line with the findings in the meta-analysis and are summarized in online Supplementary Table S5. 


\section{Kidney \\ Blood Pressure \\ Research}

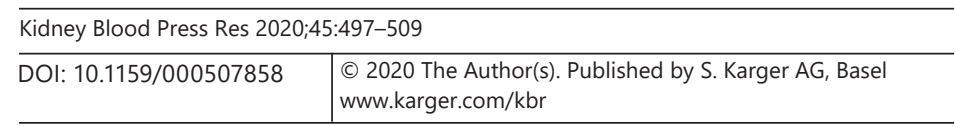

He et al.: Value of NGAL for DKD Diagnosis

\section{Discussion}

As a common complication of diabetes, DKD has become the primary cause of end-stage renal disease in developed countries. Early diagnosis and effective delay of renal impairment are of great significance in the improvement of the survival rate and quality of life of diabetic patients. According to KDOQI [14], the diagnosis of DKD is based on the presence of microalbuminuria and the reduction in eGFR. However, microalbuminuria may not be a sensitive and specific predictor for DKD, as only approximately $30 \%$ of patients with microalbuminuria develop overt nephropathy after 10 years of follow-up [25]. Furthermore, approximately $30-45 \%$ of T2DM patients with nephropathy associated with decreased eGFR do not exhibit an increase in UACR ( $>30 \mathrm{mg} / \mathrm{g}$ ), defined as normoalbuminuric DKD. On the other hand, early renal impairment in diabetes patients is usually accompanied by ultrafiltration, and the reduction in eGFR appears relatively late. In the progression of diabetic renal impairment, inflammation and arteriosclerosis induced by metabolic and hemodynamic pressure lead to endothelial dysfunction and tubulointerstitial injury. Endothelial dysfunction progresses to albuminuria, and tubulointerstitial injury leads to an increase in renal tubular markers, suggesting that tubulointerstitial and glomerular injuries may occur as parallel processes [24].

NGAL is one of the most promising tubular markers for acute and chronic kidney disease. We systematically reviewed studies on the diagnostic accuracy of NGAL for DKD. The results indicated that sNGAL and UNGAL showed good diagnostic precision for DKD. For sNGAL, the pooled sensitivity and specificity were $0.79(0.60-0.91)$ and $0.87(0.75-0.93)$, respectively. For UNGAL, the pooled sensitivity and specificity were $0.85(0.74-0.91)$ and $0.74(0.57-0.86)$, respectively.

An ideal tubular marker of DKD should possess several properties in addition to a good classification ability: (a) good correlations with albuminuria and renal function deterioration; (b) a progressive increase from micro- to macroalbuminuria; and (c) presence in normoalbuminuric patients and usefulness for early detection of diabetic renal impairment [4]. Two studies have found that NGAL is positively correlated with albuminuria and negatively correlated with eGFR, suggesting that NGAL is correlated with renal involvement and can be used as a marker for DKD grading $[24,48]$. In a study on T2DM patients and healthy controls, Fu et al. [7] demonstrated that NGAL increased significantly in the 4 groups from healthy controls to diabetic patients with normoalbuminuria, microalbuminuria, and macroalbuminuria.

The role of tubular markers in the early diagnosis of diabetic renal impairment depends on whether the change in concentration precedes the appearance of microalbuminuria. In a study by Garg et al. [48], UACR was positively correlated with UNCR in both prediabetes and diabetes patients. ROC analysis showed that the AUCs of UNCRs for detecting microalbuminuria in prediabetes and diabetes were 0.974 and 0.956 , respectively. In our meta-analysis, 4 studies demonstrated the value of NGAL in screening normoalbuminuric diabetes. The combined AUCs of sNGAL and UNGAL were 0.973 and 0.983 , respectively. This will shed light on the hypothesis that tubulointerstitial injury may occur before glomerular injury, and NGAL may be a useful marker in the initial phase of DKD.

In a recent prospective cohort study [49], baseline UNCR was significantly higher in patients with type 2 diabetes with early renal decline (defined as a decrease in the eGFR of $>30 \%$ from baseline) than in non-decliners. However, the corresponding AUC was 0.54 , which was lower than the AUCs of UACR (0.75), kidney injury molecule-1 (0.68), and monocyte chemoattractant protein-1 (0.70). Similarly, despite the lack of consistent definitions and reference standards for meta-analyses, the diagnostic value of NGAL for renal decline was quite different in the 3 articles we included. The absence of a positive finding potentially reflects the role of NGAL in tubulointerstitial impairment in late but not early renal decline. 
On the other hand, NGAL may exist as a 25-kDa monomer, a 45-kDa disulfide-linked homodimer, or a 135-kDa heterodimer (when it is covalently conjugated with matrix metalloproteinase-9) in serum or urine specimens [50]. The association between NGAL and renal decline may be better illuminated by specific monomer assays, since NGAL complexes $>100$ $\mathrm{kDa}$ contribute variably to total immunoreactivity and possibly involve proteins from nonrenal cell types [51]. In general, further work on standardized definitions and large-scale longitudinal studies are needed.

Potential sources of heterogeneity in the present analysis included heterogeneity in methodological quality, classification of diabetes (T1DM or T2DM), target population (microalbuminuric or micro- and macroalbuminuric diabetes), optimal cutoff value, sample capacity, country, and race. Furthermore, tubular markers are sensitive to the conditions of their measurement and storage. Of the eligible studies, various assays (including ELISA, a chemiluminescent immunoassay, and a turbidimetric inhibition immunoassay) were adopted to detect NGAL, which would bring bias $[4,5]$. The choice of antibodies exerts great influence on the diagnostic accuracy because several molecular forms of NGAL exist. In addition, when specimens are stored at $-20^{\circ} \mathrm{C}$, some degradation might occur due to a higher temperature.

There were several limitations to our study. First, we used UACR and eGFR as reference standards to indicate that NGAL was useful for DKD diagnosis. More prospective studies are needed to explore its predictive value for a renal function decline over time. Second, as mentioned above, there was significant heterogeneity between our chosen studies in terms of methodological differences and biological effects. Through bivariate random-effect and subgroup analyses, the limited number of identified studies did not allow for the investigation of how the test accuracies varied between studies. Third, the clinical or patient-relevant utility of a diagnostic test was evaluated using the likelihood ratios to calculate post-test probability. The LR+ and LR- of sNGAL in our meta-analysis were 6.00 and 0.24 , respectively. The LR+ and LR- of uNGAL were 4.00 and 0.23 , respectively. In this sense, only the LR+ of sNGAL reached the level generally accepted in clinical significance (LR+ $>5$ or LR $-<0.2$ ). This indicates that sNGAL is more suitable for the confirmation of DKD but has a limited role in exclusion. The primary limitation of tubular markers is their low independent predictive value for eGFR decline and proteinuria development [4]. The combination with other clinical indicators may improve the diagnostic accuracy for DKD, and more research is needed in this field.

\section{Conclusion}

Overall, NGAL is a potentially useful classifier for DKD [6, 7] and also shows potential diagnostic value for normoalbuminuric diabetes. More focused trials are needed to further this theory and identify the optimal cutoff value.

\section{Acknowledgment}

We would like to thank Hui Wang and Dan Lu for providing language help.

\section{Statement of Ethics}

Our study adhered to the Preferred Reporting Items for Systematic Reviews and MetaAnalyses (PRISMA) guidelines [9] and the meta-analysis of observational studies in epidemiology (MOOSE) guidelines [10]. Institutional approval and patient consent were not necessary. 
Kidney
Blood Pressure
Research

\begin{tabular}{l|l}
\hline Kidney Blood Press Res 2020;45:497-509 \\
\hline DOI: 10.1159/000507858 & $\begin{array}{l}\text { @ 2020 The Author(s). Published by S. Karger AG, Basel } \\
\text { www.karger.com/kbr }\end{array}$ \\
\hline
\end{tabular}

He et al.: Value of NGAL for DKD Diagnosis

\section{Disclosure Statement}

No potential conflict of interest was reported by the authors.

\section{Funding Sources}

This work was supported by the National Natural Science Foundation of China (No. 31970857).

\section{Author Contributions}

P.H., M.B., J.H., C.D., S.S., and C.H. designed the study with input into the study protocol from all authors. P.H., J.H., and C.D. performed the statistical analyses. P.H., M.B., S.S., and C.H. drafted the manuscript, and all authors assisted with revising it critically for important intellectual content. All authors contributed to the interpretation of the data and approved the final version. C.H. is the guarantor of this work.

\section{References}

1 Saran R, Li Y, Robinson B, Ayanian J, Balkrishnan R, Bragg-Gresham J, et al. US Renal Data System 2014 Annual Data Report: Epidemiology of Kidney Disease in the United States. Am J Kidney Dis. 2015 Jul;66(1 Suppl 1):S1-305.

2 Satirapoj B, Aramsaowapak K, Tangwonglert T et al. Novel tubular biomarkers predict renal progression in type 2 diabetes mellitus: a prospective cohort study. J Diabetes Res. 2016;2016:3102962.

3 Satirapoj B. Tubulointerstitial Biomarkers for Diabetic Nephropathy. J Diabetes Res. 2018 Feb;2018:2852398.

4 Zeni L, Norden AG, Cancarini G, Unwin RJ. A more tubulocentric view of diabetic kidney disease. J Nephrol. 2017 Dec;30(6):701-17.

5 Alter ML, Kretschmer A, Von Websky K, Tsuprykov O, Reichetzeder C, Simon A, et al. Early urinary and plasma biomarkers for experimental diabetic nephropathy. Clin Lab. 2012;58(7-8):659-71.

6 Liu F, Yang H, Chen H, Zhang M, Ma Q. High expression of neutrophil gelatinase-associated lipocalin (NGAL) in the kidney proximal tubules of diabetic rats. Adv Med Sci. 2015 Mar;60(1):133-8.

7 Fu WJ, Xiong SL, Fang YG, Wen S, Chen ML, Deng RT, et al. Urinary tubular biomarkers in short-term type 2 diabetes mellitus patients: a cross-sectional study. Endocrine. 2012 Feb;41(1):82-8.

8 Bolignano D, Lacquaniti A, Coppolino G, Donato V, Fazio MR, Nicocia G, et al. Neutrophil gelatinase-associated lipocalin as an early biomarker of nephropathy in diabetic patients. Kidney Blood Press Res. 2009;32(2):91-8.

9 Stewart LA, Clarke M, Rovers M, Riley RD, Simmonds M, Stewart G, et al.; PRISMA-IPD Development Group. Preferred Reporting Items for Systematic Review and Meta-Analyses of individual participant data: the PRISMA-IPD Statement. JAMA. 2015 Apr;313(16):1657-65.

10 Stroup DF, Berlin JA, Morton SC, Olkin I, Williamson GD, Rennie D, et al. Meta-analysis of observational studies in epidemiology: a proposal for reporting. Meta-analysis Of Observational Studies in Epidemiology (MOOSE) group. JAMA. 2000 Apr;283(15):2008-12.

11 Marathe PH, Gao HX, Close KL. American Diabetes Association Standards of Medical Care in Diabetes 2017. J Diabetes. 2017 Apr;9(4):320-4.

12 Olson DE, Rhee MK, Herrick K, Ziemer DC, Twombly JG, Phillips LS. Screening for diabetes and pre-diabetes with proposed A1C-based diagnostic criteria. Diabetes Care. 2010 Oct;33(10):2184-9.

13 Chinese Diabetes Society(CDS). Chinese Guidelines for the Prevention and Treatment of Type 2 Diabetes Mellitus (The 2013 edition). Chin J Diabetes Mellitus. 2014;22(8), https://doi.org/https://doi.org/10.3969/j. issn.1006-6187.2014.08.027.

14 National Kidney Foundation. KDOQI Clinical Practice Guideline for Diabetes and CKD: 2012 Update. Am J Kidney Dis. 2012 Nov;60(5):850-86.

15 Kirsztajn GM, Filho NS, Draibe SA, Netto MV, Thomé FS, Souza E, et al. [Fast reading of the KDIGO 2012: guidelines for evaluation and management of chronic kidney disease in clinical practice]. J Bras Nefrol. 2014 Jan-Mar;36(1):63-73. Portuguese.

16 The Microvascular Complications Group of Chinese Diabetes Association. Expert consensus on the prevention and treatment of diabetic nephropathy (The 2014 edition). Chin J Diabetes Mellitus. 2014;6(11):792-801.

17 Whiting PF, Rutjes AW, Westwood ME, Mallett S, Deeks JJ, Reitsma JB, et al. QUADAS-2: a revised tool for the quality assessment of diagnostic accuracy studies. Ann Intern Med. 2011;155(8):529-36. 
He et al.: Value of NGAL for DKD Diagnosis

18 Swets JA. Measuring the accuracy of diagnostic systems. Science. 1988 Jun;240(4857):1285-93.

19 Macaskill P, Gatsonis C, Deeks J, Harbord R, Takwoingi Y. Chapter 10: Analysing and Presenting Results. In: Deeks JJ, Bossuyt PM, Gatsonis C, editors. Cochrane handbook for systematic reviews of diagnostic test accuracy version 1.0. The Cochrane Collaboration; 2010. Available from: http://srdta.cochrane. org/.

20 Sueud T, Hadi NR, Abdulameer R, Jamil DA, Al-Aubaidy HA. Assessing urinary levels of IL-18, NGAL and albumin creatinine ratio in patients with diabetic nephropathy. Diabetes Metab Syndr. 2019 Jan - Feb;13(1): 564-8.

21 Liu L, Zhang HF, Yang HX, et al. EGFR, urinary NAG and serum NGAL levels in patients with type 2 diabetic nephropathy and their significance. Zhonghua Linchuang Yishi Zazhi. 2019;47(01):52-5.

22 Żyłka A, Dumnicka P, Kuśnierz-Cabala B, Gala-Błądzińska A, Ceranowicz P, Kucharz J, et al. Markers of Glomerular and Tubular Damage in the Early Stage of Kidney Disease in Type 2 Diabetic Patients. Mediators Inflamm. 2018 Aug;2018:7659243.

23 Vijay S, Hamide A, Senthilkumar GP, et al. Utility of urinary biomarkers as a diagnostic tool for early diabetic nephropathy in patients with type 2 diabetes mellitus. Diabetes Metab Syndr. 2018;12(5):649-652.

24 Kaul A, Behera MR, Rai MK, Mishra P, Bhaduaria DS, Yadav S, et al. Neutrophil gelatinase-associated lipocalin: as a predictor of early diabetic nephropathy in type 2 diabetes mellitus. Indian J Nephrol. Jan-Feb 2018;28(1): 53-60.

25 Motawi TK, Shehata NI, ElNokeety MM, El-Emady YF. Potential serum biomarkers for early detection of diabetic nephropathy. Diabetes Res Clin Pract. 2018 Feb;136:150-8.

26 Wang QH, Chen L, Peng L. Diagnostic value of serum miRNA-21, Smad1 combined with urinary NGAL for early diabetic nephropathy. Zhejiang Pract Med. 2018;23(05):321-3.

27 Zhong YQ, Zeng XB, Ye ZD. Diagnostic value of serum NGAL and amyloid A levels in diabetic nephropathy. J Guangdong Med University. 2018;36(04):411-3.

28 Chen WC, Li L, He JY. Analysis on the value of urine NGAL, MCP-1 and RBP in the diagnosis of early type 2 diabetic nephropathy. Clin Med Eng. 2018;25(06):803-4.

29 Zeng XF, Lu DX, Li JM, Tan Y, Li Z, Zhou L, et al. Performance of urinary neutrophil gelatinase-associated lipocalin, clusterin, and cystatin $\mathrm{C}$ in predicting diabetic kidney disease and diabetic microalbuminuria: a consecutive cohort study. BMC Nephrol. 2017 Jul;18(1):233.

30 Dayem SA, Bohy AE, Hamed M, Ahmed S. Follow Up of Value of the Intrarenal Resistivity Indices and Different Renal Biomarkers for Early Identification of Diabetic Nephropathy in Type 1 Diabetic Patients. Open Access Maced J Med Sci. 2017 Mar;5(2):188-92.

31 Yang HX, Zhang HF, Liu L, et al. The value of serum NGAL, TRF, hs-CRP and SF in the diagnosis of diabetic nephropathy. Shandong Yiyao. 2017;57(46):50-2.

32 Mahfouz MH, Assiri AM, Mukhtar MH. Assessment of Neutrophil Gelatinase-Associated Lipocalin (NGAL) and Retinol-Binding Protein 4 (RBP4) in Type 2 Diabetic Patients with Nephropathy. Biomark Insights. 2016 Feb; 11:31-40.

33 Hafez MH, El-Mougy FA, Makar SH, Abd El Shaheed S. Detection of an earlier tubulopathy in diabetic nephropathy among children with normoalbuminuria. Iran J Kidney Dis. 2015 Mar;9(2):126-31.

34 Assal HS, Tawfeek S, Rasheed EA, El-Lebedy D, Thabet EH. Serum cystatin C and tubular urinary enzymes as biomarkers of renal dysfunction in type 2 diabetes mellitus. Clin Med Insights Endocrinol Diabetes. 2013 Jul; 6:7-13.

35 Hosny SS, Bekhet MM, Hebah HA et al. Urinary neutrophil gelatinase-associated lipocalin in type 2 diabetes: Relation to nephropathy and retinopathy. Diabetes Metab Syndr. 2018;12(6):1019-24.

36 Shen DH, Chen JB, Yang YH et al. Significance of serum NGAL in diagnosis of extremly early type 2 diabetic nephropathy and the evaluation of its disease progression. Cardiovasc Dis Electronic J Integrated Traditional Chin Western Med. 2016;4(26):71-72+74.

37 Lacquaniti A, Donato V, Pintaudi B, Di Vieste G, Chirico V, Buemi A, et al. "Normoalbuminuric" diabetic nephropathy: tubular damage and NGAL. Acta Diabetol. 2013 Dec;50(6):935-42.

38 Bacci MR, Chehter EZ, Azzalis LA, Costa de Aguiar Alves B, Fonseca FL. Serum NGAL and Cystatin C Comparison With Urinary Albumin-to-Creatinine Ratio and Inflammatory Biomarkers as Early Predictors of Renal Dysfunction in Patients With Type 2 Diabetes. Kidney Int Rep. 2016 Oct;2(2):152-8.

39 Satirapoj B, Aramsaowapak K, Tangwonglert T, Supasyndh O. Novel Tubular Biomarkers Predict Renal Progression in Type 2 Diabetes Mellitus: A Prospective Cohort Study. J Diabetes Res. 2016;2016:3102962.

40 Yürük Yıldırım Z, Nayır A, Yılmaz A, Gedikbaşı A, Bundak R. Neutrophil gelatinase-associated lipocalin as an early sign of diabetic kidney injury in children. J Clin Res Pediatr Endocrinol. 2015 Dec;7(4):274-9.

41 Siddiqui K, Al-Malki B, George TP, Nawaz SS, Rubeaan KA. Urinary N-acetyl-beta-d-glucosaminidase (NAG) with neutrophil gelatinase-associated lipocalin (NGAL) improves the diagnostic value for proximal tubule damage in diabetic kidney disease. 3 Biotech. 2019 Mar;9(3):66.

42 Su Y, Hai QY. Study on Combined Determination Plasma NGAL, Cys C and Urinary NAG/Crea for the Diagnosis of Early Diabetic Nephropathy. Xiandai Jianyan Yixue Zazhi. 2018;33(1):99-101.

43 Dai HY, Cao Y, Wang XJ, et al. Early diagnostic value of combined serum CysC and NGAL in type 2 diabetic nephropathy [Medical Sciences]. J Nantong University. 2018;38(04):285-7.

44 Siddiqi Z, Karoli R, Kaul A, Fatima J, Varshney S, Beg MS. Evaluation of neutrophil gelatinase-associated lipocalin and cystatin C as early markers of diabetic nephropathy. Ann Afr Med. 2017 Jul-Sep;16(3):101-6. 
45 Luo P, Mao JM, Wang H, et al. The value of SDF-1, NGAL and copeptin levels in the early diagnosis of diabetic nephropathy. Jianyan Yixue Yu Linchuang. 2017;14(14):2082-5.

46 Hao LT, Han L, Zou ZJ. Study on diagnostic value of urinary NGAL, serous and urinary cystatin C in early diabetic renal diseases. Chin J Integrated Trad Western Nephrol. 2016;(3).

47 Wang YY, Zhang Y, Lu CC, et al. Clinical significance on serum NGAL, Cys C and urinary NAG combined determination in the diagnosis of diabetic nephropathy. Lab Med. 2015;30(11):1096-9.

48 Garg V, Kumar M, Mahapatra HS, Chitkara A, Gadpayle AK, Sekhar V. Novel urinary biomarkers in pre-diabetic nephropathy. Clin Exp Nephrol. 2015 Oct;19(5):895-900.

49 Nowak N, Skupien J, Smiles AM, Yamanouchi M, Niewczas MA, Galecki AT, et al. Markers of early progressive renal decline in type 2 diabetes suggest different implications for etiological studies and prognostic tests development. Kidney Int. 2018 May; 93(5):1198-206.

50 Makris K, Rizos D, Kafkas N, Haliassos A. Neurophil gelatinase-associated lipocalin as a new biomarker in laboratory medicine. Clin Chem Lab Med. 2012;50(9):1519-32.

51 Nickolas TL, Forster CS, Sise ME, Barasch N, Solá-Del Valle D, Viltard M, et al. NGAL (Lcn2) monomer is associated with tubulointerstitial damage in chronic kidney disease. Kidney Int. 2012 Sep;82(6):718-22. 